\title{
Automated Segmentation of Resistivity Image Logs Using Wavelet Transform
}

\author{
Marina Hruška • William Corea • \\ Donald Seeburger • William Schweller • \\ William H. Crane
}

Received: 11 March 2008 / Accepted: 22 June 2009 / Published online: 17 July 2009

(C) The Author(s) 2009. This article is published with open access at Springerlink.com

\begin{abstract}
We describe a wavelet-transform-based method for automated segmentation of resistivity image logs that takes into account the apparent dip in the data and addresses the problem of discriminating lithofacies boundaries from noise and intrafacies variations. Our method can be applied to borehole measurements in general, but might have an advantage when applied to resistivity image logs as it addresses explicitly the large variability in facies segments recorded with a high-resolution multiple-sensor tool. We have developed an algorithm based on this method that might outperform other existing segmentation methods in the cases of low to moderate dip. We made a detailed comparison of the segmentation from our method with the one done by a geologist to delineate different lithofacies blocks in a well drilled in a deepwater depositional environment. Our results show considerable success rates in reproducing the geologically defined lithofacies boundaries, and the generality of our procedure suggests it could also be applied to other depositional environments.
\end{abstract}

Keywords Signal processing - Deepwater depositional environment - Lithofacies · Wavelet analysis

\section{Introduction}

Estimates of specific rock properties, such as porosity or fluid saturation that are important for creating hydrocarbon reservoir models, are made based on data from well logs and core samples. They are often interpreted in terms of distinct rock units or (litho)facies, or larger units of sedimentary bodies and depositional environments. This process, however, requires a significant amount of an interpreter's time. Automated log segmentation, which automatically partitions data into regions that are locally uniform (according to some property) and distinct from adjacent ones, provides

M. Hruška ( $\varangle) \cdot$ W. Corea · D. Seeburger · W. Schweller · W.H. Crane

Chevron Energy Technology Company, 6001 Bollinger Canyon Rd., San Ramon, CA 94583, USA

e-mail: hruska@chevron.com 
an initial simplification of this task. If done in a framework that captures important and recurring features for the required interpretation, segmentation provides a more meaningful data representation and can aid subsequent analysis.

Early methods for automated well log segmentation (zonation) were classified as local or global (Davis 2002) depending on whether they identified abrupt changes in a finite moving window (e.g., abrupt changes in the mean-Webster 1973), or by breaking the whole sequence into a predetermined number of segments as distinct and internally homogeneous as possible in an iterative procedure that analyzes the within-zone variance (Gill 1970; Hawkins and Merriam 1973). As noted by Webster, performance of local methods depends on the variability of the original sequence and the length of the moving window. Such methods can result in an inordinate number of boundaries being found within a very variable part of the sequence. Global zonation methods might avoid this problem by specifying a suitable total number of boundaries to be identified. However, it might be difficult to provide this number a priori in a long highly variable sequence as is common for noisy log data.

Another problem in designing an automated segmentation method is posed by the often indistinct, evolving nature of sedimentary boundaries with a progressive decrease or increase in grain size. Such trends, as reflected in a gamma-ray measurement or a resistivity image, might be easier to detect by studying a signal-derived quantity that differs from those that indicate an abrupt change. An abrupt change in the signal, such as a smoothed step change, could be located where there is a modulus maximum of the first derivative of the signal (as exploited by the Canny edge detector-Canny 1986), or a zero in the second derivative of the signal (Marr and Hildreth 1980). By contrast, the location of a change in the trend would be characterized generally by a modulus maximum of the second derivative.

It has long been recognized that discrimination of edges from textures in an image depends on the scale of analysis (Mallat 1999; Rosenfeld and Thurston 1971). Physiological studies of the visual cortex (Julesz 1981), suggesting the existence of an internal spatial-frequency representation that is capable of preserving both local and global information, have inspired several approaches to texture analysis using banks of Gabor filters of different scale and orientation tuning. However, decompositions of this type are computationally quite intensive and outputs are not mutually orthogonal resulting in a significant correlation between textural features. These problems can be avoided if one uses a wavelet transform, which provides a precise and unifying framework for the analysis and characterization of a signal at different scales (Daubechies 1992; Grossman and Morlet 1984).

Multiscale methods (Vermeer and Alkemade 1992) and wavelet methods in particular (Prokoph and Agterberg 2000; Robail and Rabiller 1998; Robail et al. 2001) have recently been applied to the segmentation of open-hole logs. Apart from the rapid processing that such techniques offer geologists, these methods have indicated a new possibility in automated stratigraphic analysis: the hierarchical ordering of segment boundaries. These methods were applied to a gamma-ray log (e.g., Robail and Rabiller 1998), where the smallest desired partitions were limited by the resolution of the measurement. However, modern resistivity image logs with resolutions of 3-5 cm and multiple sensors around the borehole can resolve both fine changes in lithology (such as thin beds of sand and shale) and the geometry of the rock strata (indicating 
the depositional environment). The algorithm of Vermeer and Alkemade (1992) was successful in finding partitions that have sharp-edged boundaries between them and a small degree of variation (within partitions). Their method starts partitioning on a coarse scale and progresses to finer scales by inserting more boundaries into segments where the log values depart significantly from a linear fitting function. For a $\log$ containing sufficient information to discern a thinly-bedded section, partitioning would stop only when each thin bed is given its own segment. Robail and Rabiller (1998), on the other hand, used wavelet thresholding to avoid obtaining an overly segmented log, choosing a data-specific threshold value.

We present a wavelet-transform-based method for the segmentation of resistivity image logs and other well logs that takes into account the apparent dip in the data. We address the problem of discerning lithofacies boundaries from noise and intrafacies variations in the following way. First, we smoothed the data with a Savitzky-Golay filter (Press et al. 1992). Then we chose an orthogonal wavelet family with an optimally small support and performed wavelet thresholding of the smoothed data with the average magnitude of the wavelet transform coefficient moduli as a threshold (at a scale comparable to that characteristic of lithofacies change). Thus, our threshold is an internally calculated parameter that does not need to be operator-adjusted when processing another well. In addition, our method has an advantage in cases of known moderate to high apparent dip due to the local dip adjustment of the data. We also present test results from a well drilled in a deepwater depositional environment, whereby we compared in detail how well our method reproduced the boundaries identified manually in $600 \mathrm{~m}$ of borehole resistivity images. These results show that our method is well suited to a geologic interpretation of lithofacies in a deepwater depositional environment, whereas the generality of our procedure suggests that the same algorithm could be applied to resistivity image segmentation in other environments with minor modifications (if any).

\section{Methodology}

\subsection{Wavelet Transform}

A wavelet family of functions is generated from a function (mother wavelet) $h(x)$ by the operation of dilations and translations (Daubechies 1992)

$$
h_{a, b}(x)=|a|^{-1 / 2} h\left(\frac{x-b}{a}\right), \quad a, b \in \mathbf{R}, a \neq 0 .
$$

If the function $h(x)$ satisfies an "admissibility condition" (Daubechies 1992, p. 7; Mallat 1999, p. 82), functions $f(x) \in L^{2}(\mathbf{R})$ (where $L^{2}(\mathbf{R})$ is the Hilbert space of measurable, square-integrable functions) can be represented uniquely (up to a constant) by functions

$$
(W f)(a, b)=|a|^{-1 / 2} \int d x h\left(\frac{x-b}{a}\right) f(x) .
$$


If $a$ and $b$ are allowed to vary continuously, $(W f)(a, b)$ represents a continuous wavelet transform.

For the ease of mathematical presentation, wavelets are often introduced as functions with a zero average (Mallat 1999, p. 5),

$$
\int d x h(x)=0
$$

and the input functions, $f(x)$, are assumed to belong to the $L^{2}(\mathbf{R})$ space of functions.

If one restricts the values of parameters $a$ and $b$ in (2) to a discrete sublattice with a dilation step $a_{0}>1$ and a translation step $b_{0} \neq 0$, one obtains the discrete wavelet transform $(T f)$

$$
(T f)_{m n}=\left\langle h_{m n}, f\right\rangle=\left|a_{0}\right|^{-1 / 2} \int d x h\left(a_{0}^{-m} x-n b_{0}\right) f(x),
$$

where $\left\langle h_{m n}, f\right\rangle$ are the wavelet coefficients and $m$ and $n$ are whole numbers. Computations are performed over discrete signals. However, in the limit when sampling intervals decrease towards 0 , a theory of continuous functions gives asymptotic results which are precise enough to understand the behavior of discrete algorithms (Mallat 1999, p. 9). In the context of detecting sharp changes in signal, it is notable that the continuous wavelet transform in (2) is proportional to the first derivative (in shift variable) of the signal $f$ smoothed on the scale $a$ and evaluated at the point $b / a$ (Mallat and Zhong 1992). Smoothing is performed with a smoothing function $\theta(x)$ that satisfies $h(x)=d \theta(x) / d x$, and the scale $s=a$ of smoothing is defined from dilation as $h_{s}(x)=(1 / s) h(x / s)$. When $\theta(x)$ is Gaussian, detection of local modulus maxima of $W f$ (as a function of the wavelet shift) corresponds to a Canny edge detection (Mallat 1999, p. 176). It can be proved under rather general conditions that the absence of local modulus maxima of a function's wavelet transform in a given region implies that this function has a certain degree of regularity (Lipshitz) in this region (Mallat 1999, p. 177). Detection of rapid changes in signal is simplified under these conditions to an identification of the modulus maxima of the signal's wavelet transform.

Choosing which wavelet family to use is an area of active research (Kane et al. 2001). Sometimes the choice is motivated by empirical tests (Robail et al. 2001). In our choice of a wavelet family, we were striving for simplicity of the algorithm and a sparse representation for resistivity image logs.

A Gaussian smoothing function is useful for detecting distinct contours in natural images (Vermeer and Alkemade 1992). In natural textures, however, sharp changes in signal occur close together; it is therefore of interest to represent them by wavelets of finite small support (a region of points that the wavelet function maps to non-zero values). Resistivity image logs that represent fine bed laminations are often closer to natural textures than other open-hole logs.

Stephane Mallat constructed a fast orthogonal wavelet transform algorithm (Mallat 1999, p. 255) for decomposing a signal into a series of approximated versions at scales that differ successively by factors of 2 (Fig. 1). Apart from the appeal of its simplicity, this algorithm uses an orthogonal wavelet representation that can efficiently approximate (with few non-zero wavelet coefficients) particular classes of 


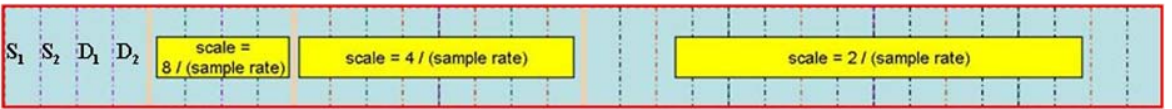

Fig. 1 The structure of the wavelet coefficient vector in Mallat's algorithm. The second half of the vector contains wavelet coefficients for the scale that is twice the sample spacing. The second quarter of the vector contains those on the scale that is four times larger than the sample spacing. The scale increases in the second eighth to twice that, and so on. The first two components $S_{1}, S_{2}$ contain the so-called "mother function coefficients" (see Press et al. 1992) and the third and fourth $\left(D_{1}, D_{2}\right)$ are the wavelet coefficients at the coarsest scale (half the length covered by the sample). Higher scales are represented with fewer components

functions (Mallat 1999, p. 241) and is useful for many applications, including the detection of sharp changes in the signal.

A function $f$ has few non-negligible wavelet coefficients if most of the fine-scale (high resolution) wavelet coefficients are small. This depends mainly on the regularity of $f$, the number of vanishing moments, $p$, of the wavelet $h$ (i.e., $\int_{-\infty}^{\infty} t^{k} h(t) d t=0$ for $0 \leq k<p$ ), and the size of its support. For a function $f$ that is well approximated in the neighborhood of a point by a polynomial of degree $k(0 \leq k<p)$, representation by a wavelet family with $p$ vanishing moments means that the fine scale wavelet coefficients in that neighborhood are near zero. The support size, on the other hand, directly affects the size of neighborhood around an irregularity of $f$ in which fine scale coefficients are non-zero. The size of support of a function and the number of its vanishing moments are generally independent, but specific properties of orthogonal wavelets imply that a wavelet of $p$ vanishing moments has a support size of at least $2 p-1$. Daubechies' wavelets are optimal in the sense that they have a minimum size of support for a given number of vanishing moments (Mallat 1999, p. 244). We used the implementation of Mallat's algorithm by Press et al. (1992) to calculate the wavelet transform with Daubechies' wavelet with $p=2$ vanishing moments. Coefficients of this wavelet at scale $s$ are related to the second derivative of the weighted average of this function with a smoothing function dilated by $s$. For an example, see Fig. 2.

\subsection{Relevant Scales, Thresholding and Consistency Across Sensor Pads}

We studied the behavior of wavelet coefficients at several scales as consecutive powers of 2 in the units of sample spacing. The coefficients at scales in the range of about 0.3 to $0.7 \mathrm{~m}$ stood out where there was a facies transition (see Fig. 3) that occurred visually over the region of similar extent. We exploited this property to construct our algorithm for identifying lithofacies transitions.

For data smoothing, we applied Savitzky-Golay filters. These filters are often used to make the relative widths and heights of spectral lines visible in noisy spectrometric data (Press et al. 1992, p. 650). Preserving the width of different lithofacies blocks without too much blurring of their boundaries is of particular interest to us. Smearing that would usually result from most smoothing operations would also reduce the wavelet coefficients of the resulting function and make the recognition of boundaries more difficult. We used the symmetric Savitzky-Golay filter of order 4 and a width of 128 samples (corresponding to about $0.5 \mathrm{~m}$ of data), which is capable of resolving 
(1)! (2) $:\left(2^{\prime}\right)$

(3)

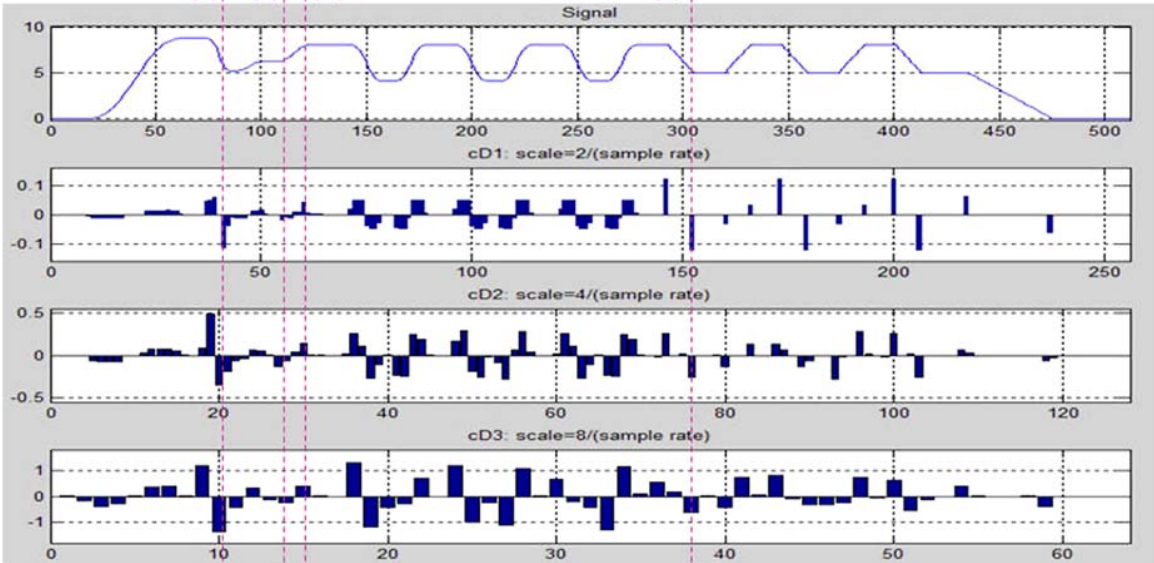

Fig. 2 (Top to bottom) A signal and its wavelet coefficients obtained with Daubechies' $p=2$ wavelet, at scales $2 /$ (sample rate), $4 /$ (sample rate) and $8 /$ (sample rate). The changes in the signal have a parabolic form up to the coordinate of 240, except for the changes marked with numbered dotted lines, which are: (1) cubic, (2) and $\left(2^{\prime}\right)$ exponential. Another change marked with a dotted line is numbered (3) and has a discontinuous first derivative. Notice the correspondence of the locations of wavelet coefficient modulus maxima with the locations of sharpest change in the first derivative (i.e., modulus maxima of the second derivative). For each parabolic change in the signal, notice how the wavelet coefficients are of the same magnitude locally (constant second derivative)

peaks down to about $0.5 \mathrm{~m}$ width. Such filtering reduces the peaks in the signal which are of smaller width, such as noise and intrafacies variation.

Thresholding methods assume that the underlying signal in the data is stronger than the noise distortions and thus will give rise to larger wavelet coefficients. Since thresholding replaces the wavelet coefficients below a certain threshold by zeros, the signal reconstructed from the modified wavelet coefficients would be a less noisy version of the original one. In addition, by choosing a threshold larger than the wavelet coefficients of intrafacies variation, we can modify the wavelet representation of the signal to reflect only the distinct changes between facies. We used the method of uniform (or scale-specific) wavelet thresholding (Donoho and Johnstone 1994), whereby we used the scale-specific average of modulus maxima of wavelet coefficients as our threshold. As noise tends to increase the magnitude of wavelet coefficients on average (Chang et al. 2000), certain more gradual changes between facies could result in wavelet coefficients below the average for the original data but (when smoothed) above the average for smoothed data. Those changes would still be recognized by our method. It is for their preservation that the initial smoothing is desirable prior to thresholding.

We applied the analogous procedure to open-hole logs (e.g., gamma-ray logs) and found it useful for confirming major stratigraphic boundaries. This result was in agreement with Robail and Rabiller (1998) who used a data-specific threshold instead. Segmentation of a gamma-ray log side-by-side with segmentation of a resistivity image $\log$ is useful where sensitivity of the microresistivity measurement to lithology is impaired, e.g., in wet sands. 


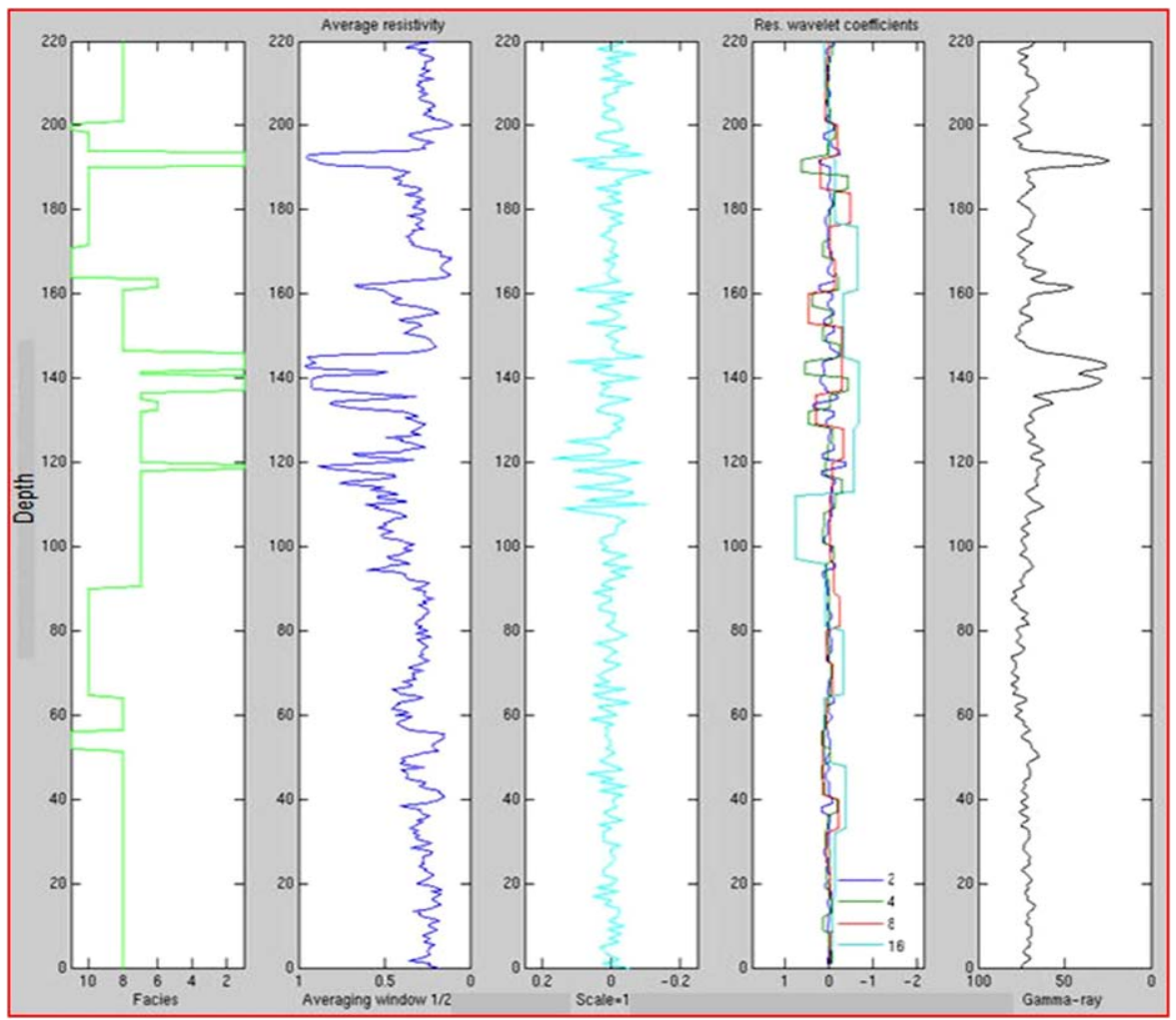

Fig. 3 Importance of wavelet coefficients on scales comparable to those of geologic facies change for the identification of lithofacies boundaries. The wavelet coefficients for two relevant scales are plotted in the third column (for scale $s$ ) and the dark blue line in the fourth column (for scale $2 s$ ), respectively, where $s$ is the unit of length used for all plots in this figure $(30.48 \mathrm{~cm}=1 \mathrm{ft})$. The first graph from the left represents a geological interpretation of facies that signifies boundaries as a change in facies code; these boundaries are the ones we desire to reproduce in an automated analysis. Note the generally increased activity of the wavelet coefficients on scale $s$ in the areas near boundaries (and somewhat in the facies block of type "7" (signifying a bedded facies)

In addition, certain geological features might result in an uneven response from the measurement device's sensors located around the borehole. An example of the layout of a resistivity imaging device is given for the Schlumberger $\mathrm{OBMI}^{\mathrm{TM}}$ one in Fig. 4. The device has 20 sensors around the borehole distributed evenly among four sensor pads. Wavelet coefficients of signals from different sensors might give large local maxima of magnitude (indicating a facies transition) at slightly different depths. This happens when sedimentary strata are strongly tilted relative to the plane in which sensors are placed (moderate to large dip) or when one of the pads has a poor contact with the borehole wall in parts of the borehole. Boundaries of tilted beds need to be recognized even though they are not distinguishable on all sensor pads at the given depth. However, not every sharp change occurring on only one sensor pad is a geologic boundary. We imposed a restriction, the "sensor-consistency criterion" or "voting criterion", to report only the boundaries that occurred around the given 


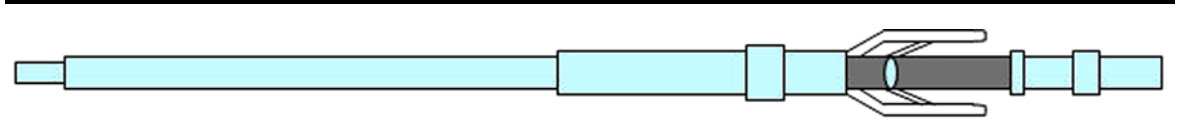

a.
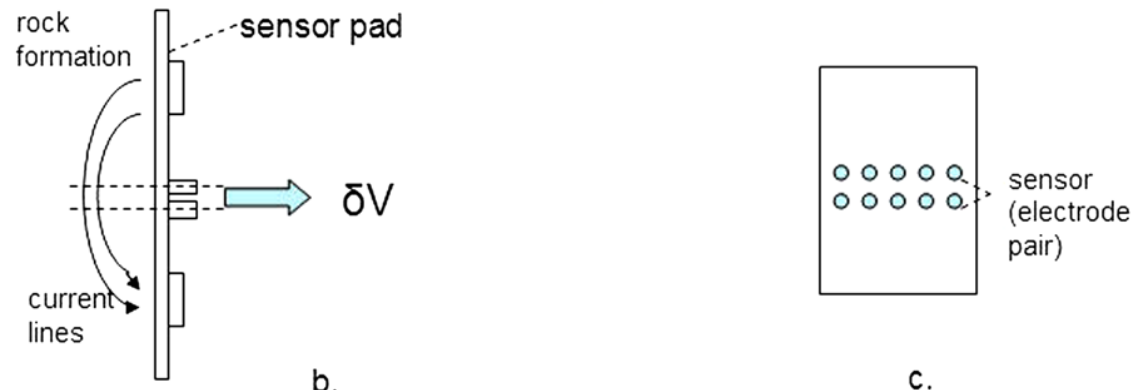

b.

c.

Fig. 4 An example of a borehole resistivity imaging device, Schlumberger OBMI ${ }^{\mathrm{TM}}$ : (a) the device includes an elongated shaft from which a number of arms (members) extend out; (b) each arm includes a sensor pad which is pressed against the borehole wall to obtain a close contact with the rock formation; (c) the sensor pad includes multiple electrode pairs (sensors) to measure a voltage drop $\delta V$ (and resistivity indirectly) in (b) for each pair when current is passed into the formation from current injector electrodes on the same pad

depth on at least as many sensor pads as an integer threshold (the sensor-consistency input parameter); a majority of sensor pads was usually desired. This ensures that a region of poor contact on a single pad will not be reported as a stratigraphic bed, and it suffices to recognize most boundaries at low dip. However, a bed boundary at high dip might appear on images from different sensor pads around the borehole at locations far apart along the vertical. To ensure that a majority of sensor pads indicates a transition at the same depth (with some reasonable accuracy), the signals recorded by different sensors around the borehole need to be processed to form a signal array (an image) that has no apparent dip (i.e., the original image needs to be adjusted such that the beds and transitions appear horizontal).

\subsection{The Local Dip Adjustment}

To extract the apparent dip, it is desirable to consider the similarity of sensor traces over a long enough interval to identify stratigraphic features. However, the interval should not be so long as to limit identification of regions with rapidly varying dip, and it should not miscorrelate a real feature to an artificial one created by noise. We computed the offsets of individual sensor signals that corresponded to the plane of the feature crossing the well at the orientation of the extracted apparent dip. These were the shifts needed to be applied to the signals to align them locally, to make beds appear perpendicular to the borehole (Fig. 5).

The important point for segmentation is how the offsets applied to the sensor signals are used to make the bedding appear perpendicular to the borehole axis. One possibility is to stretch uniformly (and interpolate) certain parts of the signal and compress (and downsample) others. Such a procedure would lead to smoothing of some boundaries and sharpening others, which would affect the identification of true 


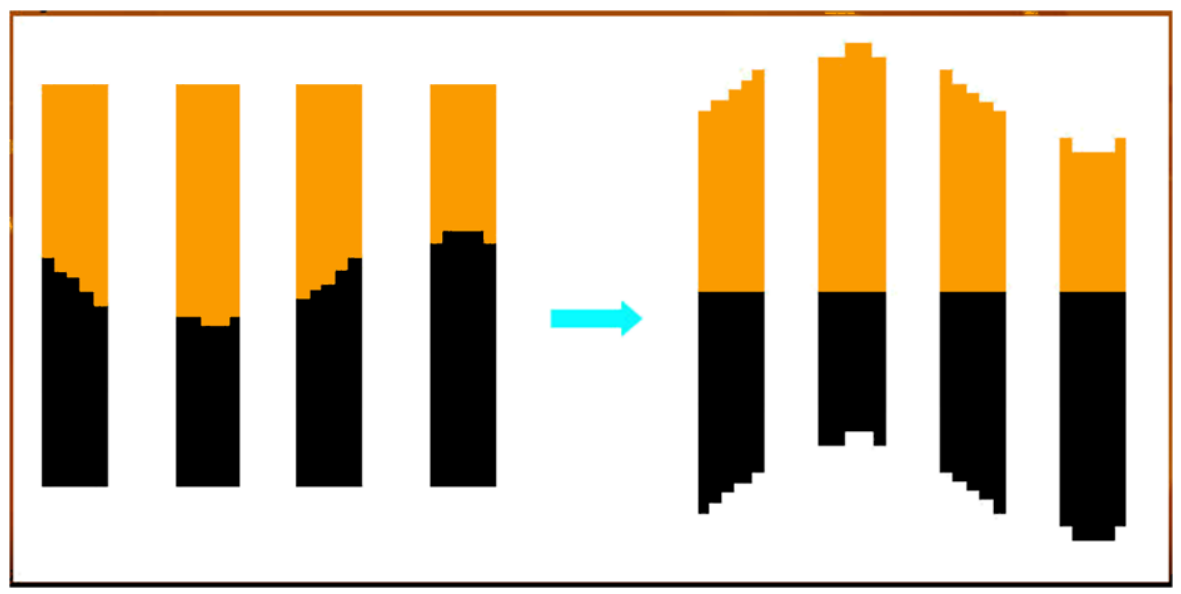

Fig. 5 Local dip adjustment for each sensor in an image formed from a four-pad device with five sensors on each pad. Alternatively, local adjustment can be implemented on pad-averages (the local dip adjustment discussed in the text)

boundaries in noise or where there is less variation. Rather than this, we used the advantage of a fast wavelet algorithm. We computed the fast wavelet transform on each sensor-pad image (except for the reference-pad one) shifted appropriately by a different dip-derived depth offset for every depth sample at the scale of interest (which was comparable to the scale of facies change). The pad shift is the average of individual sensor offsets for that pad; it is found from the dip calculation for the dip at the given depth. This is why we call our procedure a local adjustment for dip. To keep the start and end of all shifted sensor signals at the same depth and for the application of the fast wavelet transform, which requires the data sequence length to be a power of two, we omitted some of the data at the end of the sequence in the direction of shift. A gap that opened up at the other end was filled with a constant value equal to that of the last data point for simplicity. This procedure slightly enlarges the regions near the beginning and end of the data sequence where there are generally edge effects due to edge discontinuities (Mallat and Zhong 1992).

Once the wavelet transform had been performed on smoothed data that was locally adjusted for dip (dip at given depth), wavelet thresholding was done on all pads with regard to the average of modulus maxima at the scale of interest. The voting criterion was applied to the modulus maxima of the wavelet coefficients at this depth, to indicate whether there was a transition at this sample depth according to wavelet coefficients at this scale. (This procedure can be applied to several scales of interest, which are powers of two when expressed in terms of the sample spacing.) Then this procedure was repeated for the next depth sample.

The final results regarding the depths of transitions were obtained by comparing the transitions from two scales: $s$ and $2 s$, with $s=30.48 \mathrm{~cm}(1 \mathrm{ft})$. The discrete wavelet transform (3) was sampled only in increments equal to the scale, so the accuracy with which transitions were located was limited by the scale of consideration. For any scale $s_{1}$, larger than the scale $s / 2=15.24 \mathrm{~cm}(1 / 2 \mathrm{ft})$, we used the interval center of the first increment of $s / 2$ (from $2 s_{1} / s$ locations of the scale $s / 2$ wavelet co- 
efficients for the given $s_{1}$ increment) as the location of the scale $s_{1}$ wavelet coefficient. Consequently, we used correction offsets (in the direction of increasing depth) for the bed boundary location indicated by a modulus maximum at a given scale: $s / 4$ for scale $s$, whereas for the scale $2 s$ the correction is $3 s / 4$. (These offsets are not needed in the alternative notation which uses centers of $s_{1}$-scale intervals for the locations of wavelet coefficients.) However, care must be taken when combining indicators from two scales for the same bed boundary. We have noticed the correspondence of local maxima of positive coefficients (or minima of negative coefficients) at scales $s$ and $2 s$ when at the same sample depth. In this case a transition was most often centered at a distance $s / 2$ below. Also, there was a correspondence between the modulus maxima for scales $s$ and $2 s$ when the $2 s$ scale transition was at the sample depth located a distance $s$ prior to the scale $s$ transition. The correction offset for the transition in this case was $s / 4$ - the same as in the case of a single modulus maximum occurring at scale $s$. We considered that the maxima of the opposite-sign wavelet coefficients related to different transitions, and treated them independently. With these adjust-

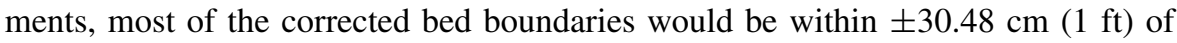
those reported by a geologist.

The procedure to determine the depths of transitions can be refined further by tracing the modulus maxima from these scales to finer scales (Mallat 1999, p. 176).

\section{The Resistivity-Image (and Other) Log Segmentation Algorithm (SegWave)}

For resistivity image logs, our "SegWave" algorithm can be summarized as follows:

(1) Adjust the data sampling in such a way that the relevant length scale $s$ for facies change (e.g., $0.3 \mathrm{~m}$ ) corresponds to a power of two in terms of the sampling interval (as appropriate for scales in the dyadic wavelet transform);

(2) Run the apparent dip and sensor offsets calculation with an appropriate correlation window;

(3) Store average sensor pad shifts at intervals of unit length scale for later local dip adjustment;

(4) Smooth resampled and pad-averaged data with a symmetric Savitzky-Golay filter of order 4 and width of 128 (valid for a $1 / 4$ to $1 / 2 \mathrm{~cm}$ sampling interval) with a routine modified from Press et al. (1992);

(5) Apply the wavelet transform to smoothed data with Daubechies' wavelets of $p=2$ vanishing moments (with a routine modified from Press et al. 1992), and find the averages of wavelet coefficient modulus maxima for scales $s$ and $2 s$ to use for wavelet thresholding;

(6) Perform the following set of operations at each sample depth at the two scales and skip the first two steps if no dip adjustment is desired:

- shift the whole pad data (except on the reference pad) according to recorded pad shifts for this depth mark;

- perform the fast wavelet transform on shifted pads;

- for each pad check how many modulus maxima are above the threshold;

- if there are at least as many maxima at this depth for a given scale as the input integer pad-consistency parameter-e.g., 3 for the Schlumberger $\mathrm{OBMI}^{\mathrm{TM}}$ in Fig. 4, store this depth and scale; 
(7) Compare these stored depths from two scales to filter out repetitions and to report corrected transition depths.

For simplicity, the first sampling adjustment step in the case of the Schlumberger $\mathrm{OBMI}^{\mathrm{TM}}$ data sampled at a rate of $1.97 \mathrm{~cm}^{-1}$ (60 per $\mathrm{ft}$ ) can be done by repeating every 15 th data point to provide 64 samples at the interval of interest for facies changes, of length $s=30.48 \mathrm{~cm}$. Artifacts introduced in this way are equivalent to an additional source of noise, which in those cases where smoothing is useful, will often be much smaller than the noise already present (see Press et al. 1992, p. 654).

To find modulus maxima of a discrete wavelet transform, one looks for maxima of positive coefficients and minima of negative coefficients.

For the segmentation of other logs, the algorithm described above can be used in essentially the same form, but omitting detail related to multiple sensors, and adjusting the scales considered according to those on which a facies change can be observed on these logs. Lower resolution of these measurements results in a more spread-out character of facies transitions, and a more pronounced response in wavelet coefficients at a larger scale than for resistivity image logs.

\section{Example}

We tested our "SegWave" algorithm on data from a deepwater depositional environment.

SegWave was run with and without the local dip adjustment on about $622 \mathrm{~m}$ of resistivity image data from one well. The well had been analyzed previously by a geologist who identified 217 transitions in about $600 \mathrm{~m}$ of geological data of reasonable quality. We obtained the following results (Fig. 6):

(1) With the local dip adjustment, whereby dip was calculated with a $91.44 \mathrm{~cm}(3 \mathrm{ft})$ correlation window, 375 boundaries were identified. Of those, 160 were within $\pm 30.48 \mathrm{~cm}$ ( $1 \mathrm{ft}$ ) from the boundaries identified by the geologist, i.e., $74 \%$ of the geologist's 217 boundaries were identified automatically. The remaining 215 boundaries identified by the program were not considered significant by the geologist.

(2) Without the local dip adjustment, 157 boundaries were identified correctly to within $\pm 30.48 \mathrm{~cm}$ ( $1 \mathrm{ft}$ ) from the geologist's boundaries, i.e., $72 \%$. Among these 157 correct boundaries, 148 were also recognized when the algorithm was run with the local dip adjustment. By combining the two runs, with and without the local dip adjustment, we would extract $157+(160-148)=169$ correct boundaries out of 217 identified by the geologist, achieving recognition of $78 \%$ of the latter boundaries. Some of the boundaries not identified when adjusting for dip might have been found with a more accurate calculation of the dip in the regions of rapidly changing or large dips.

In both cases, the percentage of the geologist's boundaries identified correctly was less between similar facies of low sand content, but much greater, up to $95 \%$, between facies with a very different sand content. Thus, missed boundaries in our case were most often those between similar, non-reservoir facies which are not always distinguished by geologists. Lowering the threshold in the algorithm resulted in the 


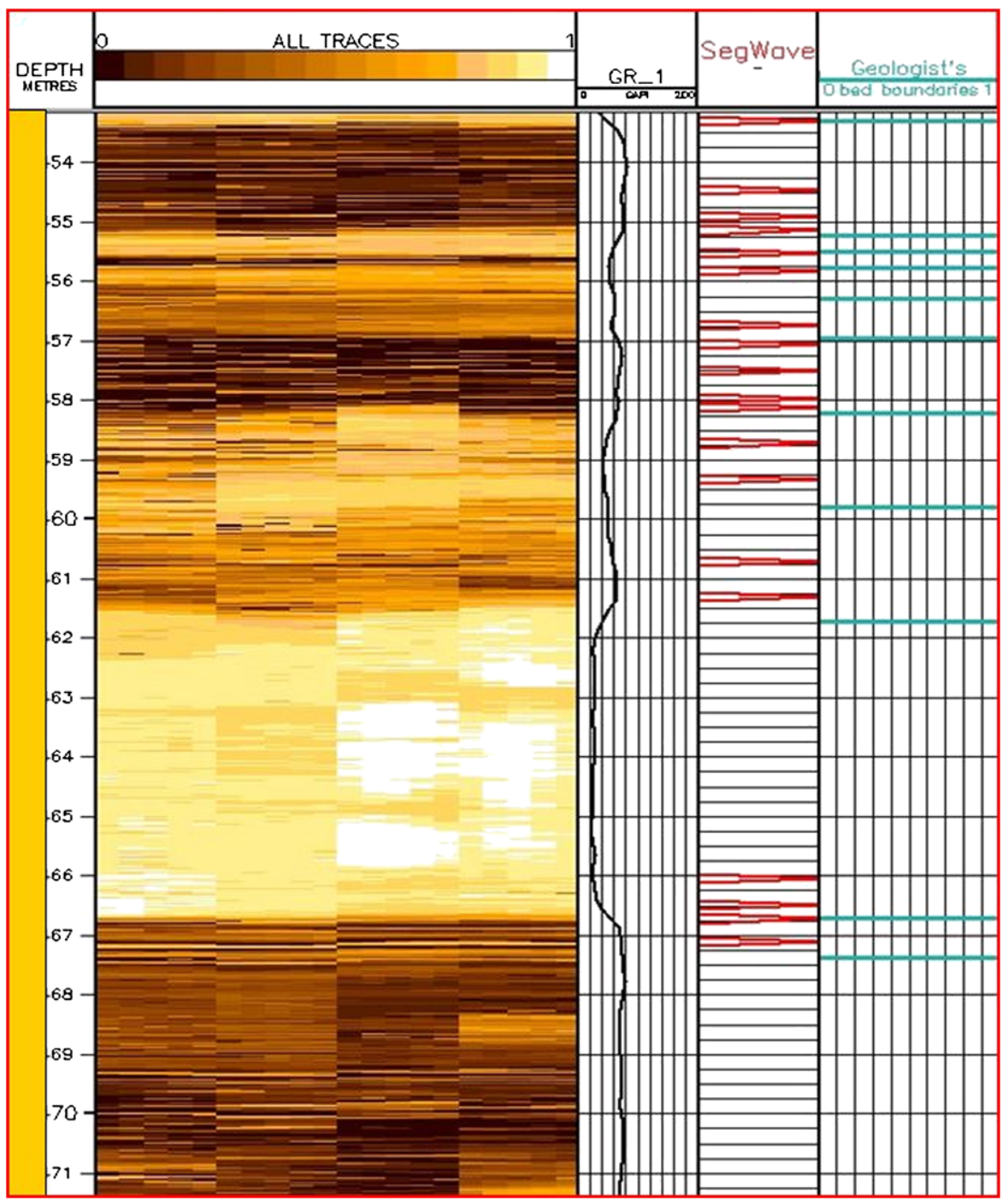

Fig. 6 Results (in the fourth column, labeled "SegWave") of segmenting the Schlumberger OBMI TM resistivity data (in the column "all traces") by our algorithm. The gamma-ray data are shown in the column "GR_1". Notice that, out of 11 geological boundaries (with their depths given in the column "Geologist's bed boundaries"), 8 have been correctly identified to a precision of $\pm 30.5 \mathrm{~cm}(1 \mathrm{ft})$, with 11 spurious boundaries reported. Note that due to a lower resolution of the gamma-ray measurement, a segmentation of only the gamma-ray data would not resolve that there are two boundaries around $55.3 \mathrm{~m}$ and might miss also the one at $67.4 \mathrm{~m}$

automatic recognition of more of these boundaries, but this could lead to the segmentation becoming too fine. Our rates of successful boundary identification with a segmentation that was as dense as that done by the geologist were satisfactory for practical resistivity image interpretation. Furthermore, the method allows several general refinements which we discuss next. 


\section{Discussion and Conclusion}

In this research, we developed and tested an algorithm for segmenting well-logs (and resistivity-image logs in particular) into lithofacies blocks. We also resolved several technical problems in the analysis of petrophysical and geologic data by exploiting the well-established properties of the wavelet transform, and by investigating the specific behavior of wavelet coefficients of lithologic boundaries relative to noise. As a result of the local dip adjustment, our segmentation algorithm performed as robustly on data with a known moderate apparent dip as on horizontally bedded facies.

The local dip adjustment might become important when extending this method to more complex depositional environments with facies differentiated mainly by their texture. Our algorithm could be applied in an analogous way to texture-sensitive properties, such as derived standard deviations of resistivity data in the directions along and perpendicular to the borehole axis. Local dip flattening prior to calculating the standard deviation along beds is essential to help distinguish a bedding of non-zero dip from a chaotic facies.

The precision with which transition depths are identified can be improved further by adjusting the reference pad transition depth to correspond to the line joining the center points between troughs and peaks of a sinusoidal bed boundary image.

In matching the results of our method with those of a geologist's interpretation, we ultimately face the limitations of the sensitivity of our measurements, as do other methods of interpretation. Each physical device has a finite resolution and is sensitive only to a specific set of rock properties. Resistivity image logs are sensitive to a combination of lithology, pore structure and fluid content. Although they are more suitable for discriminating a chaotically textured facies block from a homogeneous mudstone than a gamma-ray measurement, there is a general difficulty for geologists to distinguish these facies from each other in well logs. For future development we plan to incorporate some degree of supervision into our procedure to help to overcome this problem.

Acknowledgements We thank Dean Gulick, Boqin Sun, David Wilkinson, Bill Kowalik, Gopa De, Barry Reik, Rafael Salazar-Tio and Chris Skelt, of Chevron Energy Technology Company, and Thomas Luu of the Lawrence Livermore National Laboratory, for useful discussions and comments.

Open Access This article is distributed under the terms of the Creative Commons Attribution Noncommercial License which permits any noncommercial use, distribution, and reproduction in any medium, provided the original author(s) and source are credited.

\section{References}

Canny J (1986) A computational approach to edge detection. IEEE Trans Pattern Anal Mach Intell 8(6):679-698

Chang SG, Yu B, Vetterli M (2000) Spatially adaptive wavelet thresholding with context modeling for image denoising. IEEE Trans Image Proc 9(9):1522-1531

Daubechies I (1992) Ten lectures on wavelets. Society for Industrial and Applied Mathematics, Philadelphia, Pennsylvania

Davis JC (2002) Statistics and data analysis in geology. Wiley, New York

Donoho DL, Johnstone IM (1994) Ideal spatial adaptation via wavelet shrinkage. Biometrika 81(3):425455 
Gill D (1970) Application of a statistical zonation method to reservoir evaluation and digitized-log analysis. Bull Am Assoc Petroleum Geol 54(5):719-729

Grossman A, Morlet J (1984) Decomposition of Hardy functions into square integrable wavelets of constant shape. SIAM J Math Anal 15(4):723-736

Hawkins DM, Merriam DF (1973) Optimal zonation of digitized sequential data. Math Geol 5(4):389-395

Julesz B (1981) Textons, the elements of texture perception and their interactions. Nature 290:91-97

Kane J, Herrmann F, Töksoz N (2001) Wavelet domain linear inversion with application to well logging. In: Society of Exploration Geophysicists Int'l Exposition and Annual Meeting

Mallat S (1999) In: A wavelet tour of signal processing. Elsevier Academic, San Diego, p 189

Mallat S, Zhong S (1992) Characterization of signals from multiscale edges. IEEE Trans Pattern Anal Mach Intell 14(7):710-732

Marr D, Hildreth E (1980) Theory of edge detection. Proc R Soc Lond, Ser B 207(1167):187-217

Press WH, Teukolsky SA, Vetterling WT, Flannery BP (1992) Numerical recipes in C: the art of scientific computing. Cambridge University Press, Cambridge

Prokoph A, Agterberg FP (2000) Wavelet analysis of well-logging data from oil source rock, egret member, offshore eastern Canada. AAPG Bull 84(10):1617-1632

Robail F, Rabiller P (1998) Détection des ruptures dans les signaux diagraphiques. French Patent N9̊98/12876

Robail F, Rabiller P, Schulbaum L (2001) Sedimentary bodies identification using the phase coefficients of the wavelet transform. In: Society of Petrophysicists and Well Log Analysts 42nd Annual Logging Symposium, Paper VV, pp 1-12

Rosenfeld A, Thurston M (1971) Edge and curve detection for visual scene analysis. IEEE Trans Comput C 20:562-569

Vermeer PL, Alkemade JAH (1992) Multiscale segmentation of well logs. Math Geol 24(1):27-43

Webster R (1973) Automatic soil-boundary location from transect data. Math Geol 5(1):27-37 